\title{
CALM: Complex Adaptive System (CAS)-Based Decision Support for Enabling Organizational Change
}

Richard M. Adler, PhD DecisionPath, Inc. rich@decpath.com

David J. Koehn, PhD

DJ Koehn Consulting Services, Inc. koehndj@msn.com

Guiding organizations through transformational changes such as restructuring or adopting new technologies is a daunting task. Such changes generate workforce uncertainty, fear, and resistance, reducing morale, focus and performance.

Conventional project management techniques fail to mitigate these disruptive effects, because social and individual changes are non-mechanistic, organic phenomena.

CALM (for Change, Adaptation, Learning Model) is an innovative decision support system for enabling change based on CAS principles. CALM provides a low risk method for validating and refining change strategies that combines scenario planning techniques with "what-if" behavioral simulation. In essence, CALM "test drives" change strategies before rolling them out, allowing organizations to practice and learn from virtual rather than actual mistakes. This paper describes the CALM modeling methodology, including our metrics for measuring organizational readiness to respond to change and other major CALM scenario elements: prospective change strategies; alternate futures; and key situational dynamics. We then describe CALM's simulation engine for projecting scenario outcomes and its associated analytics. CALM's simulator unifies diverse behavioral simulation paradigms including: adaptive agents; system dynamics; Monte Carlo; event- and process-based techniques. CALM's embodiment of CAS dynamics helps organizations reduce risk and improve confidence and consistency in critical strategies for enabling transformations. 


\section{Introduction}

Guiding organizations through transformational change is a daunting task. Examples of change include downsizings, mergers, and adopting new enterprise software systems or technology platforms. Transformational changes drive fundamental shifts in personal and organization ways of thinking and doing business. As such, they disrupt the status quo, forcing managers and workers out of their comfort zones, altering their mental models, and conflicting with established behavior patterns, processes, and cultural norms. Reactions resemble the body's immune response: organizations and individuals resist change and act to maintain prior "equilibrium" conditions, lowering morale, focus, and performance. Even if desired changes are implemented successfully, major challenges remain to sustain changes: absent ongoing vigilance, organizations tend to revert back to older, familiar behaviors and attitudes. In short, change, once effected, must be institutionalized to endure.

Businesses and government agencies report high failure rates in navigating, much less sustaining, transformations, even after spending considerable sums on change management consultants [1]. Diagnostic surveys reveal low levels of employee trust in management, and chronic dissatisfaction with working conditions. Unless these underlying root causes are addressed directly, management interventions to anticipate and mitigate the disruptive effects of transformational change will continue to fail.

This paper describes an innovative methodology for enabling and sustaining change called CALM ${ }^{\mathrm{TM}}$ (for Change, Adaptation, and Learning Model). CALM provides a low risk method for validating and refining change strategies. In essence, CALM "test drives" change strategies before rolling them out, allowing organizations to practice and learn from virtual rather than actual mistakes. Equally important, CALM allows organizations to monitor change strategies as they are being executed, and perform mid-course corrections as necessary. CALM enables change teams to sense and respond at the right moment, just as expert sailors tack quickly in response to small changes in the wind. CALM thereby reduces risk and improves confidence and consistency in transformational strategies.

\section{Transformational Change}

\subsection{Related Work}

The literature on change management is extensive, produced primarily by business school professors and management consultants [2]. Authors typically describe the disruptive forces and behaviors that they observe afflicting organizations facing major change, and then prescribe process-oriented methodologies designed to mitigate those problems. Kotter [3], for example, proposes the following process model. He argues that these eight phases are jointly necessary (but individually insufficient) to guide organizations through major changes and to sustain them.

- Initiate change (by defining a sense of urgency and business case for change) 
- Build a coalition of change agents

- Formulate vision

- Communication and educate

- Empower others to act

- Create short-term wins

- Consolidate and further change

- Institutionalize change

Broadly speaking, change phenomenologies, while valuable for general anticipatory purposes, are purely qualitative and not directly actionable. We find additional problems with prescriptive process methodologies and how they are applied:

- Change management programs tend to focus at a tactical level, addressing a specific pending transformation rather than strategically, on generalized organizational preparedness and receptivity to continuous change

- Change process models apply the same mechanistic techniques that were developed to manage business and technology projects (e.g., scheduling, resource allocation) to the complex behaviors displayed by organizations and individuals facing disruptive changes - uncertainty, inertia, resistance

- Until recently [4], the literature effectively ignored decades of research on "new sciences" that focus expressly on modeling the kinds of social and personal behaviors observed in change situations, such as CAS, system dynamics, and stochastic methods. This latter literature, however, is not directly actionable.

\subsection{CALM's Contribution: “Test Driving” Change Strategies}

CALM addresses these problems by providing a dynamic decision support methodology. Rather than simply framing plans that encompass the eight phases of Kotter's change process, CALM introduces scenario-based situational modeling and "what-if" simulation capabilities to help organizations proactively validate and refine such plans. Specifically, CALM adds the following elements to the mix:

- A rich set of metrics designed to measure an organization's readiness to change

- A model of the dominant environmental forces, both internal and external, that influence organizations while they attempt to change

- A framework for defining organizational strategies - or transformation plans comprised of change initiatives such as communication and compensation techniques that contribute to Kotter's archetypal phases for helping organizations and their workforces to accept and embrace significant changes

- A CAS-inspired dynamic model that estimates the (qualitative) effects of situational forces and change initiatives over time on CALM's readiness metrics

Consultants experienced with CALM facilitate meetings with teams of organizational leaders, managers and key employees to characterize the following: 
- Organizational structure, key forces, and the pending (or ongoing) change

- Estimated current organizational readiness levels and specified target levels, which, if achieved would likely ensure success

- A small number of alternate plausible futures defined by assumptions of how environmental forces, trends, and singular events might play out

- One or more prospective alternate transformation plans, composed of pre-defined initiatives from CALM's library, which incorporate projected schedules and costs.

The CALM methodology is embodied in a software system implemented using DecisionPath's ForeTell framework [4]. ${ }^{1}$ ForeTell captures these various situational and assumptive elements in model constructs called scenarios. Consultants then apply CALM's simulation engine to project the likely outcomes of prospective plans - in terms of changing readiness metrics across alternate scenarios. Finally, integrated analytics enable projected outcomes to be explored and compared, across both alternate futures and transformation plans, to identify a robust change strategy.

Intuitively, a "robust" strategy is one that produces attractive results across alternate futures. No one can predict the future reliably. The next best thing is to devise a change plan that carries a high likelihood of success regardless of which future obtains. By projecting the likely consequences of candidate plans across diverse plausible futures, CALM helps organizations identify relative strengths and weaknesses, and uncover unintended consequences. Stronger plans can then be synthesized from preceding attempts. In short, the CALM framework supports the validation of iterative refinement of strategies to increase robustness.

Change strategies, however inspired, have little value unless they are executed competently. CALM was designed to be applied after strategies have been adopted, to monitor their execution. In this mode, organizations update their scenarios periodically to reflect current conditions and progress in improving readiness. CALM then re-projects the chosen strategy against these updated scenarios. If outcomes continue to be positive (i.e., readiness metrics reach their target levels), the chosen strategy is re-validated. If not, CALM acts as an Early Warning System, helping to uncover emerging problems promptly; diagnose them; and define and validate midcourse corrections. In this post-decision "sense and respond" mode, CALM helps organizations carry out and sustain change strategies across their extended "lifecycles," despite inevitable situational changes that occur over time.

\subsection{Modeling the Behavioral Dynamics of Organizational Change}

Enabling and sustaining transformational change is a complex, extended process that is influenced by several basic dynamic drivers. First, as organizations carry out

\footnotetext{
${ }^{1}$ ForeTell is a software platform for rapidly developing and deploying critical decision support systems. It has been applied to diverse domains including counterterrorism and pandemic preparedness, competitive drug marketing strategy, IT portfolio management, and dynamic social network analysis.
} 
change initiatives, internal and external stakeholders invariably respond, adapting their behaviors to advance personal and group interests.

Second, individual and organizational attitudes and behaviors tend to evolve over time in complex, and often non-linear patterns. For example, trust, morale, focus, and acceptance typically don't build or decay continuously and smoothly; rather, they tend to jerk, stick, and accelerate or decelerate.

Finally, environments such as societies and markets evolve continually, driven by situational forces and events. In short, the target audience and the "ground" under the organization's "feet" shift continually and in ways that are difficult to anticipate. It is the complex interplay of intentional adaptive and non-linear behaviors and ongoing environmental change that causes static process models to fail.

CALM improves how transformational plans are developed, validated, and executed because it recognizes and embraces these complex dynamics of change. It applies "new science" theories such as system dynamics, CAS, and Monte Carlo methods specifically designed to model individual and social behavior patterns. Finally, it provides an iterative and interactive process for modeling and analyzing change and change strategies using these "organic" dynamic modeling techniques.

A simple analogy explains CALM's approach and differentiate it from process models such as Kotter's. Enabling and sustaining transformational change is similar to launching a rocket. Propelling a rocket into orbit requires generating sufficient over time to (1) overcome inertia and lift the rocket's mass, and (2) accelerate it to "escape" velocity for insertion into orbit despite the constant drag of gravity. If insufficient thrust is generated and maintained, the rocket will fall back to earth. In multi-stage rockets, the ignition and firing durations of booster sections must be designed precisely to produce sufficient thrust and appropriate acceleration profiles.

Similarly, change strategies must generate organizational and personal "thrust" to bring out (i.e. accept and embrace) change and sustain it by overcoming persistent conservative forces such as personal and group inertia and old behavior patterns. As in multi-stage rocket engines, the sequencing and durations of change activities must be carefully coordinated to prevent the loss of momentum and perceptions of stalled progress, which lead directly to outright failure or unraveling of transformations.

This analogy is admittedly imperfect. Designing rocket engines and launch trajectories is a well-established engineering discipline. Newton's laws of motion strictly determine the mechanical interactions of a small number of key parameters and forces. Solutions can be computed from textbooks or software programs. The same equations apply uniformly to all launch situations. They never change over time.

Organizational change clearly represents a more complex and open-ended phenomenon. It is not obvious what parameters to measure, much less what, if any 
"universal laws" govern situational dynamics. As a result, models such as CALM are qualitative rather than quantitative, and exploratory than deterministic and predictive.

Process models such as Kotter's, while valuable, provide guidance that is largely static, passive, and broadly defined: they offer no framework for thinking through the dynamics of change processes, or help in designing or selecting and assembling specific initiatives to implement the eight stages tailored to particular organizations and their specific transformational challenges.

Most organizations need more detailed guidance. Enabling change is a complex undertaking. Mistakes are inevitable. They may also be irreversible. Initiatives that fail tend to undermine stakeholder trust and confidence: management cannot simply switch strategies and try again from the same initial state. Something must be added to help organizations design and test change strategies in advance.

Our rocket analogy provides this critical missing ingredient, namely, a model for anticipating how transformational change is likely to play out in terms of empirical metrics. CALM equates Kotter's eight step model to a multi-stage rocket engine. CALM also defines an explicit dynamic model of how change initiatives (and situational forces) impact measurable organizational readiness factors. This simulation model allows CALM to project how organizations, their employees, forces, and change initiatives will interact with one another and evolve over time.

Transformation plans can thereby be validated dynamically, much as rockets can be simulated to see if they generate the required launch and thrust profiles. For CALM, sufficient "thrust" over time equates to improvements in the key readiness metrics. Reaching escape velocity corresponds to achieving target readiness values. Failure to achieve target values means that the transformation plan is unlikely to succeed.

CALM's simulation, while qualitative, provides a systematic and repeatable basis for projecting likely outcomes. Through iterative refinement (and continuous improvement over successive transformations), CALM helps organizations address change and learn how to execute better over time. The next section describes the software that embodies this methodology.

\section{Embodying the CALM Methodology in Software}

CALM organizes the process of "test driving" change strategies into three primary processes - modeling, behavioral "what-if" simulation, and analysis. Each process is supported by a software application that was developed using DecisionPath's ForeTell ${ }^{\circledR}$ decision support software platform [5], as summarized in Figure 1. 
Model

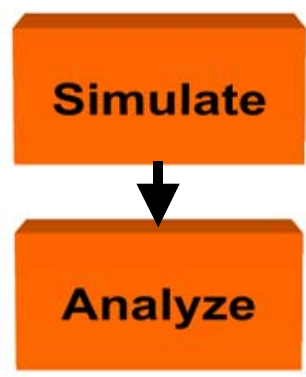

Situation: organization, environment, change Candidate change strategies $\&$ tasks

\author{
What if situation evolves along path $Y$ ? \\ What if we intervene by doing $X$ ?

\section{Explore projected outcomes (key metrics) Compare alternate decisions \& scenarios}

\subsection{Modeling}

CALM explicitly captures available knowledge about both static and dynamic aspects of situational contexts and transformations. Capturing situational dynamics is clearly critical for CALM's second task - projecting outcomes. ${ }^{2}$

CALM change models, called scenarios, are comprehensive, encompassing the following situational elements mentioned above, ${ }^{3}$ summarized pictorially in Figure 2:

- Relevant organizational units (e.g. an agency, its member departments or groups)

- The transformation at issue (e.g., new IT system technology, a reorganization)

- Current and target (goal) values for metrics that measure organizational readiness to change (and overall performance)

- Environmental forces and trends acting on the organizational unit on a continuing basis (and assumptions about how they are likely to change over the future)

- Possible events that might occur and disrupt the transformation and change plan

- Candidate transformation plans, composed of individual change initiatives

\footnotetext{
${ }^{2}$ Lacking supporting software, conventional change management methodologies address dynamics in an informal, intuitive manner, if at all. This ad hoc approach is very difficult to apply consistently, much less replicate or teach.

${ }^{3}$ These elements are implemented as a hierarchical object-oriented model: the Scenario class contains (is the parent of) Organizational Unit, which contains Change, Metric, Environmental Force, and Transformation Plan subclasses.
} 
Environmental forces include both external factors (e.g. social, political, legal, economic) and internal factors (e.g., leadership, resources). Examples of disruptive events include new legislation or regulations, changes in leadership or economic conditions.

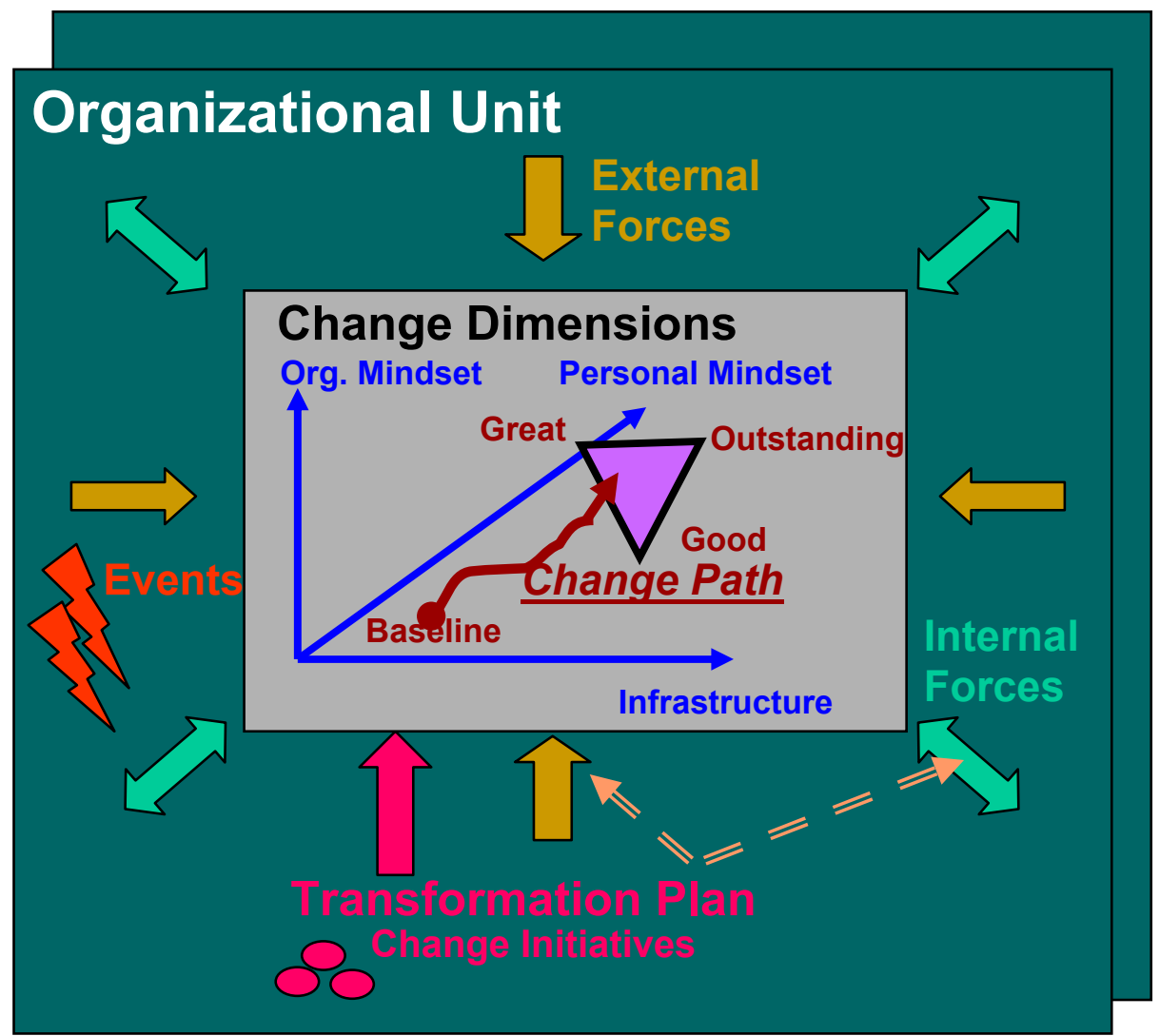

Figure 2. CALM Elements for Modeling Organizational Change

Metrics are critical for measuring an organization's initial, goal, and evolving states surrounding a transformation. Existing change methodologies focus primarily on metrics relating to business "infrastructure", which measure "physical things to do" regarding organizational technology processes, structures, and policies (e.g., functional training to use a new business software system). However, the dominant causes for transformation failures typically trace back to less obvious and less concrete organizational and workforce readiness factors, such as cultural dissonance; inadequate leadership support; poor communication; fear of change; lack of confidence; or inadequate skills or empowerment to carry out the necessary adaptations. Failure to attend to these intangible factors can result in winning the battle, but losing the war: organizations know what to do to carry out the requisite changes, but are unwilling to accept or embrace them. 
The CALM methodology measures organizational readiness to deal effectively with change across three "dimensions" - infrastructure, organizational and personal (workforce) mindsets. Each CALM change readiness dimension consists of three to six metrics, selected from the literature to reflect diverse types of transformations and associated risks. Example organizational mindset metrics include teaming, cultural coherence, and leadership change capacity. Personal mindset metrics reflect factors such as emotional resilience (emotional intelligence/personal change skills), learning capabilities, competency development and self-confidence. Infrastructure metrics include operational agility, technological upgradeability and organizational adaptability. CALM also provides metrics for "bottom-line" performance (e.g., cost competitiveness, customer satisfaction). ${ }^{4}$ All metrics are estimated on a scale of 1 to 100. Exact values are less important that the reasoning (documented by CALM) that teams use to come to consensus on them.

CALM's Transformation Plan construct exploits the Kotter process model. The eightphased plans are constructed of finer-grained initiatives or strategies, which have projected schedules and estimated costs. Incorporating costs into change plans allows CALM to support differential cost-benefit analyses of competing plans.

CALM's behavioral dynamics model projects the likely impacts on the target organization (and its readiness measures), of changes in the situational environment induced by forces, events, and the proposed change enablement strategy. CALM exploits ForeTell's dynamic architecture to project likely impacts, combining simulation techniques including complex adaptive systems (agents) [6,7], event modeling, system dynamics [8], and Monte Carlo methods [9].

Each environmental Force in CALM specifies its (assumed) causal impact. That is, it dictates how a change in magnitude (e.g., of bold new leadership, increased competition) is likely to impact the organizational performance and readiness metrics described earlier. Similarly, each change initiative (e.g., communicating vision, empowering employees to carry out change tasks) specifies how that activity is likely to impact CALM metrics over their scheduled times.

As in the real world, these effects may take time to manifest (i.e. delay or latency), have bounded durations, and display cumulative build-up (i.e., non-linear response). The data to drive these specifications comes from expert judgment and other sources (e.g., surveys, benchmarking exercises, data warehouses) that may be available. Users construct, copy and edit, and export scenarios using an intuitive graphical user interface (GUI) modeled after standard tree-based controls for viewing file systems. The Scenario Editor contains panels that depicts the entities comprising a scenario, the attributes for a selected entity, and integrated help that explains the entity type and attribute. Pop-up editors provide text, numerical slider, list and table controls for

\footnotetext{
${ }^{4}$ The CALM software allows the baseline set to be extended with additional metrics tailored to particular organizations, sectors, and changes.
} 
entering scenario data, as appropriate. All values can be annotated with comments, source, and degree of certainty, facilitating transfer and maintenance of scenarios.

For ease-of-use and speed, we are populating a library of pre-defined forces and change initiatives integrating with CALM. Each such component provides a prevalidated causal model, which can be tuned to specific organizations and contexts as required. CALM's library allows drives a "Lego ${ }^{\mathrm{TM}}$ building block" approach to building scenarios that exploits best practices expert knowledge about change strategies and their likely impacts on organizational readiness over time.

\subsection{Simulation Engine}

CALM projects situational dynamics via ForeTell's parallel discrete event simulation engine. The core engine employs an agent-based simulation paradigm: at each simulated interval (i.e. "clock tick"), this engine invokes active scenario entities in a uniform order based on the object hierarchy. Each such entity runs its type-specific behaviors. For intentional entities (i.e., goal-directed organizations or persons), actions often involve sensing internal and external state and responding according to behavioral patterns, such as stimulus-response rules.

The ForeTell engine employs a novel hybrid architecture that extends the core CAS paradigm with overlays that support other simulated dynamics, including causality (i.e., system dynamics), situational trends, events, and processes. For example, a CALM transformation plan carries out organizational change initiatives as a process comprised of scheduled tasks, the causal impact of which is realized by system dynamic productions. ${ }^{5}$ A similar embedded system dynamics model propagates the effects of CALM environmental forces on organizational readiness metrics.

ForeTell's multi-modal simulator reflects the fact that real world systems are influenced by diverse - and interacting - dynamic drivers. Uni-modal simulators frequently lead to distorted or incomplete models when their chosen dynamic paradigm is applied outside of its ideal design stance (e.g., modeling environmental interactions with agents or systems of intentional entities with system dynamics).

Users monitor and control executing scenarios through ForeTell's "dashboard" style GUI, made up of controls, gauges and time series plots of aggregate readiness metrics. Simulations can be suspended to inspect specific entities and metrics.

\subsection{Analytics}

As the ForeTell simulator runs, it logs all state changes occurring in CALM scenario entities to a database. An integrated analytics engine helps users retrieve and reduce

\footnotetext{
${ }^{5}$ ForeTell's simulator incorporates a Monte Carlo utility that allows users to define statistical distributes of scenario parameters and perform large numbers of runs (trials) in batch mode, which is useful for sensitivity analysis. It also supports dynamic social network modeling and Bayesian inference networks.
} 
this mass of data via graphical and tabular summaries to explore projected outcomes of individual CALM scenarios. More importantly, outcomes can be compared across scenarios for competing strategies and/or diverse plausible futures.

Users generate summary analytics via a simple menu-driven dynamic query GUI. Outputs include tabular reports, time series and radar plots, and frequency histograms. One summary report documents before/after metrics and percentage change, while others isolate specific dynamics (events, trends, causality) that help users understand why and when observed readiness changes occurred. ForeTell's analytics engine embeds open source math, graphics, and statistics libraries, allowing rapid extension to satisfy new analytic requirements.

\subsection{Validation and Verification}

Organizations typically require confidence building exercises before they are willing to commit to the CALM methodology. CALM employs several techniques for this purpose. First, when available, we use an organization's prior transformational projects to calibrate CALM's dynamics, by adjusting force and change initiative causal weighting factors to match historical patterns of changing readiness levels.

Secondly, the CALM methodology prescribes two types of validation exercises, called "sanity checking" and retrospective testing. In sanity checking, scenarios are constructed to depict extreme conditions and change strategies. People often lack firm intuitions about the minor variations in forces or plans. However, they typically have stronger instincts about outcomes in extreme situations.

For example, suppose employees face a change situation that entails deep pay cuts and/or layoffs. Given this context, most people would predict with confidence an outcome that includes major drops in employee morale and customer satisfaction. An organization's CALM scenarios should project outcomes that match these beliefs. They should also change gracefully as the extreme conditions are "dialed back."

Retrospective testing on past change situations represents the "acid test". Here, scenarios are created that depict the organization's state and environment at the (historical) point of decision. Next, the forces, trends and events that actually occurred through to the present are added to the scenario. Finally, the change initiatives that were undertaken are introduced. Given these inputs, CALM's scenario projections should resemble the evolution of organizational readiness metrics that actually took place.

\subsection{Conclusions}

CALM provides a disciplined framework drawn from CAS principles for modeling organizational transformations and exploring alternate change enablement strategies. CALM acts as a "robotic juggler", uniformly manipulating the diverse interacting

dynamic drivers of change that humans are cognitively unable to project mentally in a consistent manner. In essence, CALM enables organizations to practice prospective 
change strategies in a low risk virtual environment. Organizations can then learn from simulated mistakes at minimal cost, rather from real "blood on the tracks" errors that result in problems such as pervasive worker mistrust or alienated customers.

CALM can be applied over the lifecycle of extended transformations, helping organizations sense and respond to continuous situational change. CALM also supports decision-making for less radical incremental or transitional forms of organizational change.

CALM's differential analyses of alternate change strategies across diverse plausible futures are key drivers of enhanced decision-making. All significant changes involve risk: risk is unavoidable. Managing risk effectively hinges on understanding the likely costs and benefits of assuming particular risks and incurring only those risks for which the rewards are commensurate. CALM helps organizations explore these trade-offs systematically and repeatably. CALM libraries capture and disseminate best practice analyses of change and change strategies. CALM simulations also provide audit trail that enhance organizational governance, continuous learning and improvement. Thus, the CALM methodology helps organizations reduce risk and increase confidence in responding to transformational change.

\section{Bibliography}

[1] Pascale, R., Millemann, M., and Gioja, L. Surfing the Edge of Chaos, p. 12.

[2] Useful Web-based bibliographies for change management literature include: http://www.dhrm.state.va.us/training/change/resources_bibliography.html, http://www.change-management.com/bookstore.htm, http://www2.nrcan.gc.ca/es/msd/emmic/web-enemmic/index.cfm?fuseaction=subjects.subjectchange.

[3] Kotter, J.P. Leading Change: Eight Ways Organizational Transformations Fail. Harvard Business Review, March-April, 1995, 59-67.

[4] Herasymowych, M. and Senko, H., Navigating through Complexity: Systems Thinking Guide, 2nd Edition, 2002, www.mhainstitute.ca.

[5] Adler, R. M., "ForeTell: A Simulation-Based Modeling and Analysis Platform for Homeland Security Decision Support". Proceedings Second IEEE Conf. on Technologies for Homeland Security. Cambridge, MA. May, 2003.

[6] Bar-Yam, Y. Dynamics of Complex Systems, Westview Press, Cambridge, MA 1997.

[7] Epstein, J.M. and Axtell, R., Growing Artificial Societies: Social Science from the Bottom Up. Brookings Institution Press, Washington DC, 1996.

[8] Sterman, J., Business Dynamics: Systems Thinking and Modeling for a Complex World, Cambridge, MA, Irwin McGraw-Hill, 2000.

[9] Fishman, G.S., Monte Carlo: Concepts, Algorithms, and Applications, New York, Springer, 1996. 